\title{
IMPLEMENTATION OF NAIVE BAYESIAN DATA MINING ALGORITHM ON DECEASED REGISTRATION DATA
}

\author{
Dr. P. Y. Desai \\ Associate Professor, Department of Information and Communication Technology, \\ Veer Narmad South Gujarat University, Gujarat, India
}

\begin{abstract}
The use of data mining algorithm in different domains like marketing, finance, retail, cyber security, fraud detection, medical science, etc...is well known. In recent times, the data mining is also implemented for e-governance data. Normally, egovernance data are only used for Online Transaction Processing System (OLTP). However, one can use data mining algorithm to uncover hidden and new trends from e-governance data. In this paper, Naïve Bayes data mining algorithm is applied on Deceased Registration data to find hidden trends.
\end{abstract}

Key words: Deceased Registration Data, Naive Bayes Algorithm, Data Mining.

Cite this Article: Dr. P.Y. Desai, Implementation of Naive Bayesian Data Mining Algorithm on Deceased Registration Data. International Journal of Computer Engineering and Technology, 10(1), 2019, pp. 32-37.

http://iaeme.com/Home/issue/IJCET?Volume=10\&Issue=1

\section{INTRODUCTION}

The naïve bayes data mining algorithm is used to solve different types of real world problems. As per [1], the naïve bayes algorithm is used for text classification. In [2], the naïve bayes classification is used for loan risk assessment and data set of tunisian commercial bank. Their study demonstrated that classification rate was 58.66\% [2]. Reference [3] used naïve bayes algorithm for student dataset classification. Their experiments showed that naïve bayes classifier was having accuracy of $66.67 \%$ [3]. In [4], naïve baye algorithm was implemented for email spam classification problem. Their study recommends that naïve bayes algorithm is better results than support vector machine [4]. In [5], the naïve bayes algorithm was used to find credit card fraud detection. Their study showed that their accuracy was $95 \%$ using the naïve bayes algorithm [5]. Reference [6] discussed about use of the naïve bayes algorithm in text news classification. Their study showed that using naïve bayes algorithm, they were able to classify text news into four different categories [6]. In [7], naïve bayes classifier was used for prediction of cancer risk in perspective of symptoms. Their study included symptoms of lung cancer, symptoms of breast cancer, oral cancer, ovarian cancer, and ovarian cancer. Their study concluded that the naïve bayes classification can be effectively used in early 
cancer detection [7]. As per [8], naïve bayes classification is used for opining mining. Their study showed that sentiment analysis can be done based on reviews made by the users. The naïve bayes algorithm is used to classify review in positive and negative classes [8]. Similarly, the naïve bayes algorithm can also be effectively used for deceased registration data for finding hidden trends and relationship among various attributes.

\section{RESEARCH METHODOLOGY}

In this paper, Microsoft Analysis Services and Microsoft Naïve Bayes algorithm is used. The proposed research methodology is as follows:

\section{Select the Deceased Data Cube}

First, Deceased Data Cube was selected to perform the Naïve Bayes Data Mining algorithm. The Data Cube contains various dimensions and measures pertaining to Deceased Registration data.

\section{Configure parameters of Microsoft Naïve Bayes algorithm}

The algorithm parameters were set considering the Deceased Data Cube. The MAXIMUM INPUT ATTRIBUTES parameter was set to seven, as seven input attributes were considered. The MAXIMUM OUTPUT ATTRIBUTES parameter was set to one, as only one predictable attribute was considered. The MAXIMUM STATES which indicate total states of an attribute to be considered while building data mining model. This attribute was kept to default as it was difficult to find number of possible states from all data.

\section{Select the Input Attributes}

In this step various input attributes like Death Place, Age, Deceased Religion, Deceased Sex, Medical Attention Type, Year and Death Date were selected.

\section{Select the Key Attributes}

The Death Date (Date and Time Field) attribute was selected as Key attribute to uniquely indentify each row from the data cube.

\section{Select the Output Attributes}

Medical Attention Type attribute was selected as Predict attribute to find relationship among various input attributes and Medical Attention Type attribute.

\section{Generate Mining Model}

Considering steps A to E, the Naïve Bayes data mining model was generated for Deceased Registration Data Cube.

The research methodology is depicted in the Figure 1. and results are discussed in the result section. 


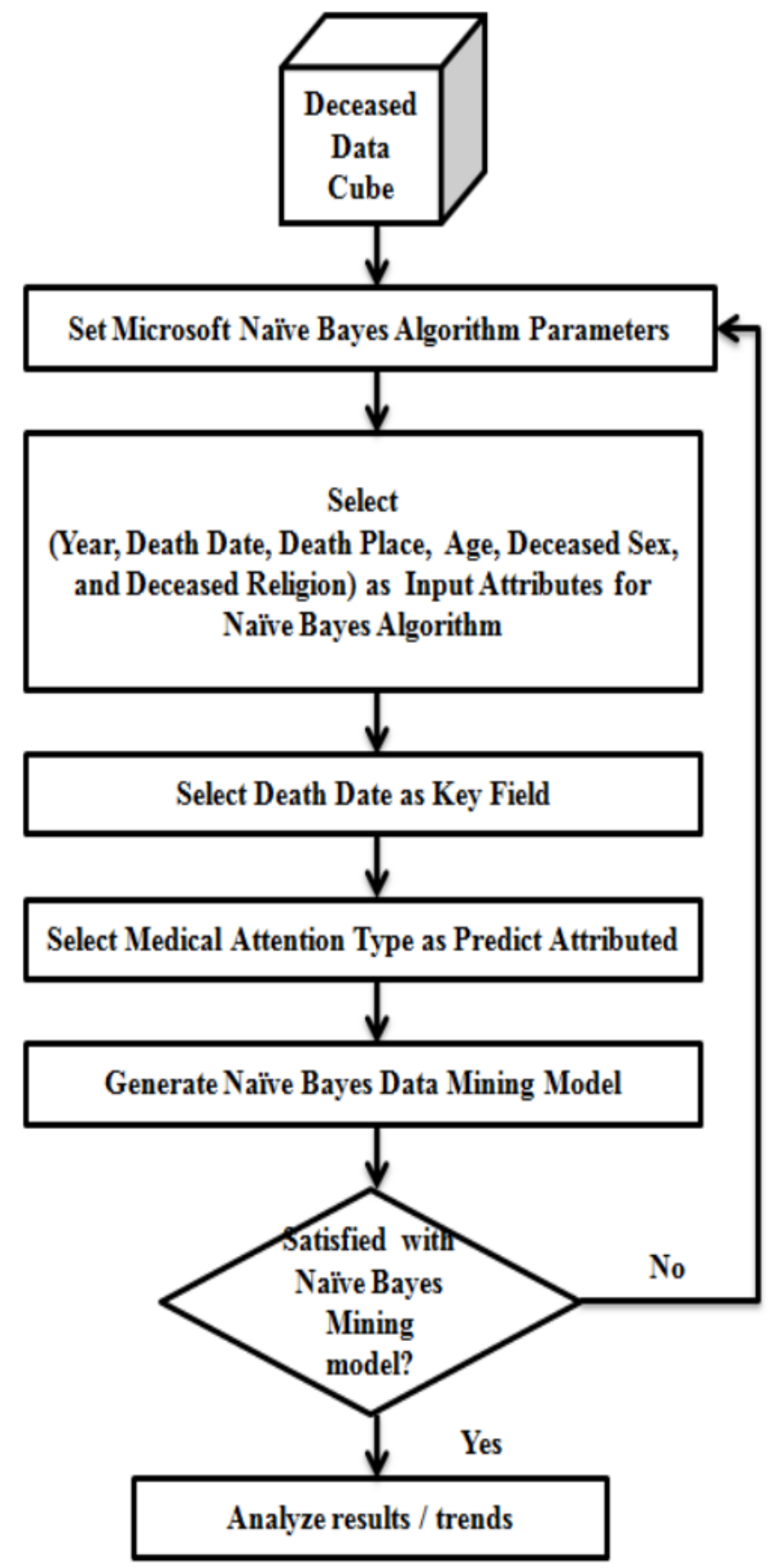

Figure 1 Proposed Methodology

\section{RESULTS}

In the Naïve Bayes Data Mining model, Medical Attention Type was kept as Predictable attribute. The Medical Attention Type has three possible values. The Medical Attention Type 1 indicates "Institutional", Medical Attention Type 2 indicates "Medical attention other than Institution" and Medical Attention Type indicates " No medical attention". 
Dr. P.Y. Desai

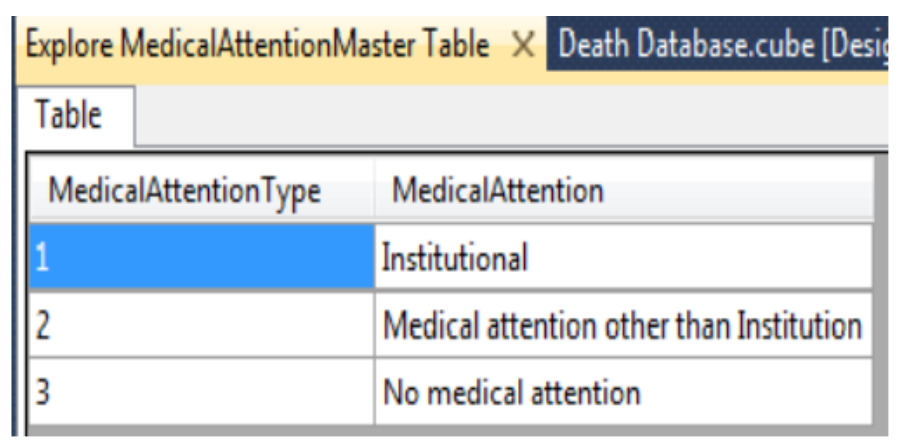

Figure 2 Medical Attention Type Output Attribute

The possible states for Religion ID input attribute is as per Figure 3.

\begin{tabular}{|c|c|}
\hline Table & \\
\hline DeceasedReligionID & DeceasedReligionName \\
\hline 1 & Boddh \\
\hline 2 & Christian \\
\hline 3 & Hind \\
\hline 4 & Hindu \\
\hline 5 & Jain \\
\hline 6 & Muslim \\
\hline 7 & Other \\
\hline 8 & Parsi \\
\hline 9 & Shikh \\
\hline
\end{tabular}

Figure 3 Religion ID Input Attribute

The result showed interesting relationship between Religion ID and Medical Attention Type attribute.

- The results indicate that for Religion $I D=6$ which is Muslim religion is having highest probability for No medical attention with probability of 0.55 . The result is shown in Figure 4 .

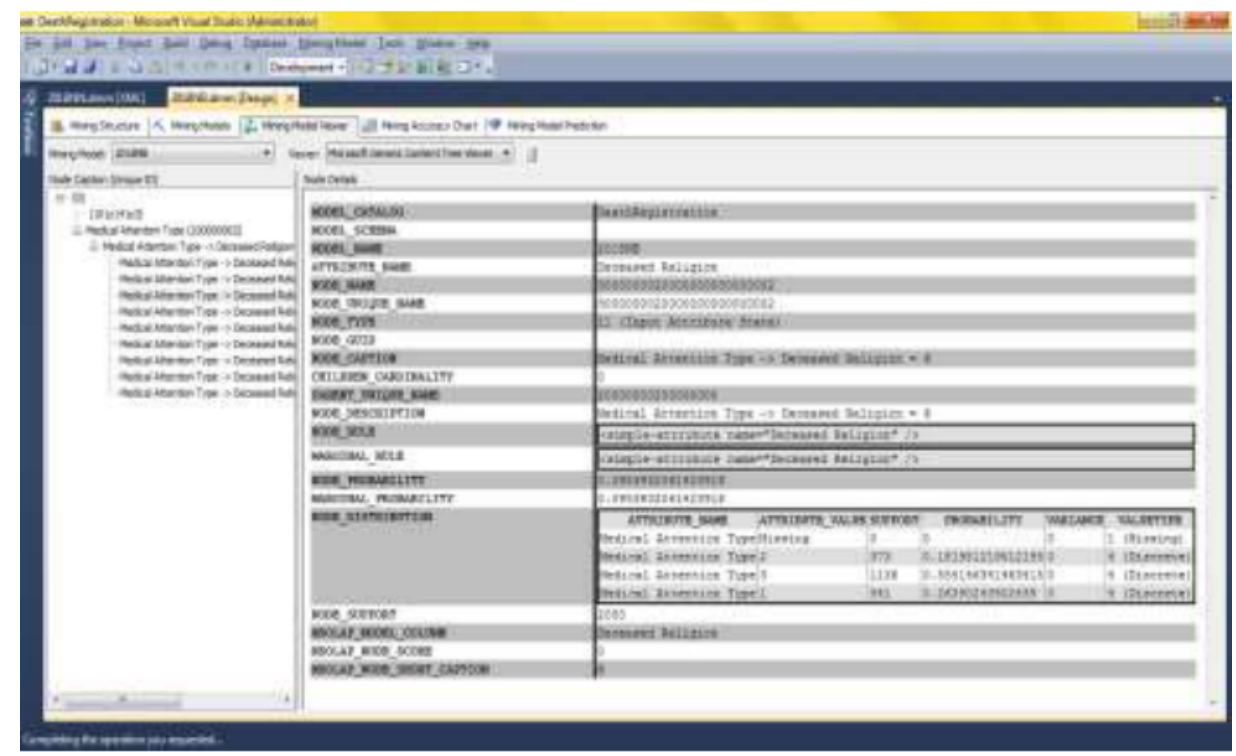

Figure 4 Result for Religion ID=6 input Attribute 
- The results showed that for Religion $\mathrm{ID}=4$ which is Hindu religion is having highest probability for No medical attention with probability of 0.65 . The result is shown in Figure 5 .

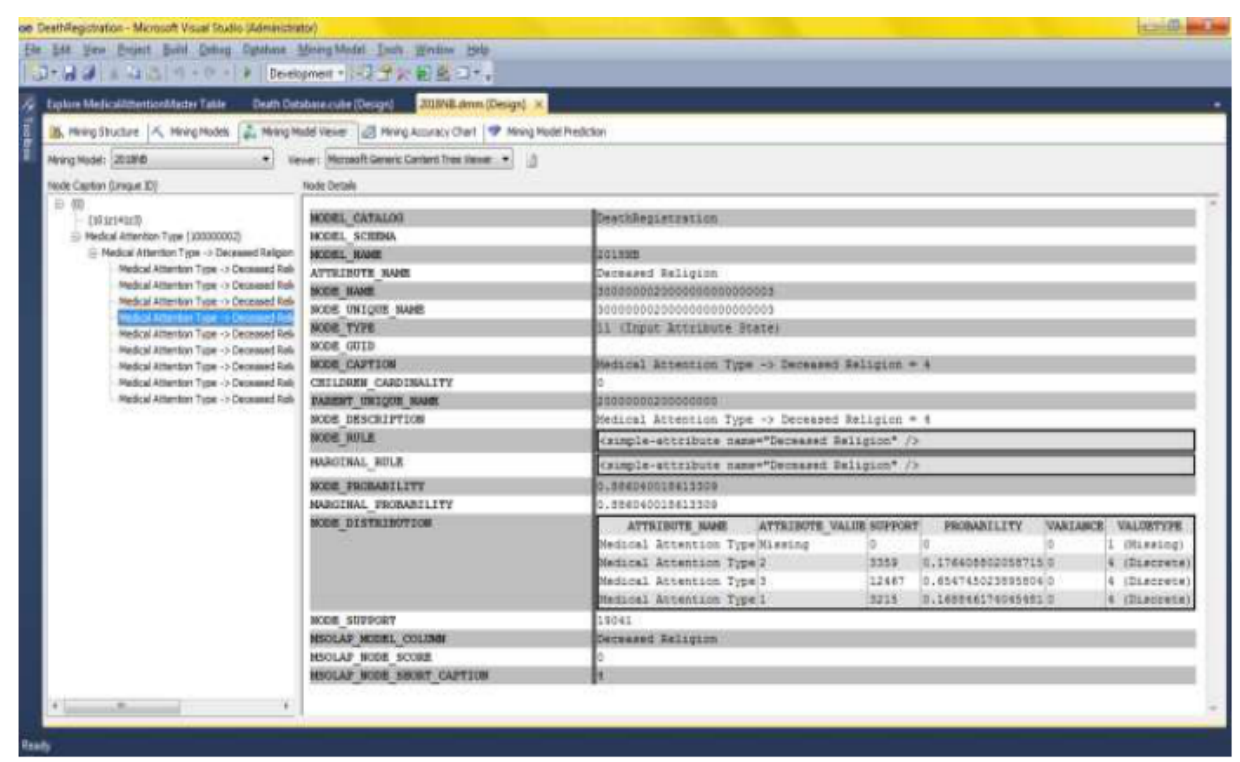

Figure 5 Result for Religion ID $=4$ input Attribute

More trends were derived from the attribute discrimination option provided in the Microsoft Analysis Services.

- As per attribute discrimination results, Religion $\mathrm{ID}=6$ which is Muslim favours Medical Attention Type $=1$, where as Religion $\mathrm{ID}=4$ which is Hindu favours Medical Attention Type $=2$. This result is shown in the Figure 6 .

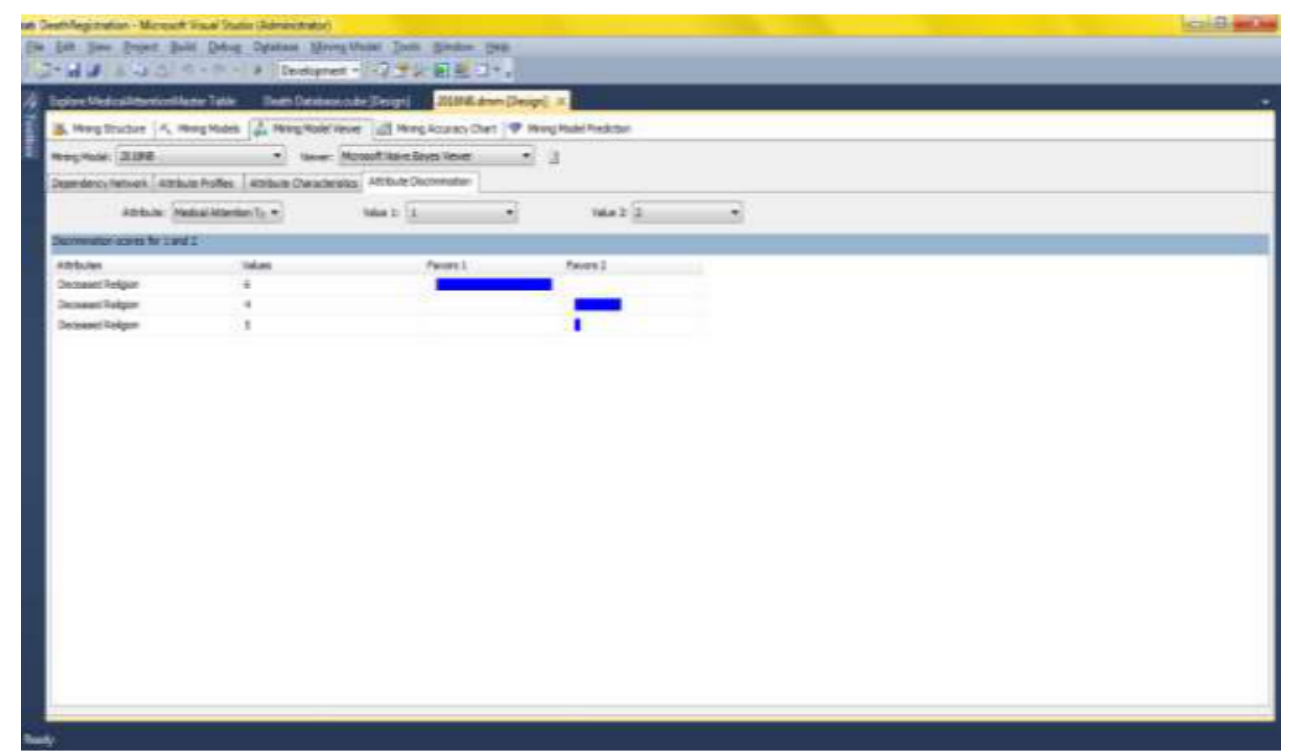

Figure 6 Result for Attribute Discrimination Medical Attention Type 1 and 2

- In other results, Religion ID $=8$ which is Parsi favours Medical Attention Type $=2$ whereas Religion ID $=4$ which is Hindu favours Medical Attention Type $=3$. The result is shown in the Figure 7. 


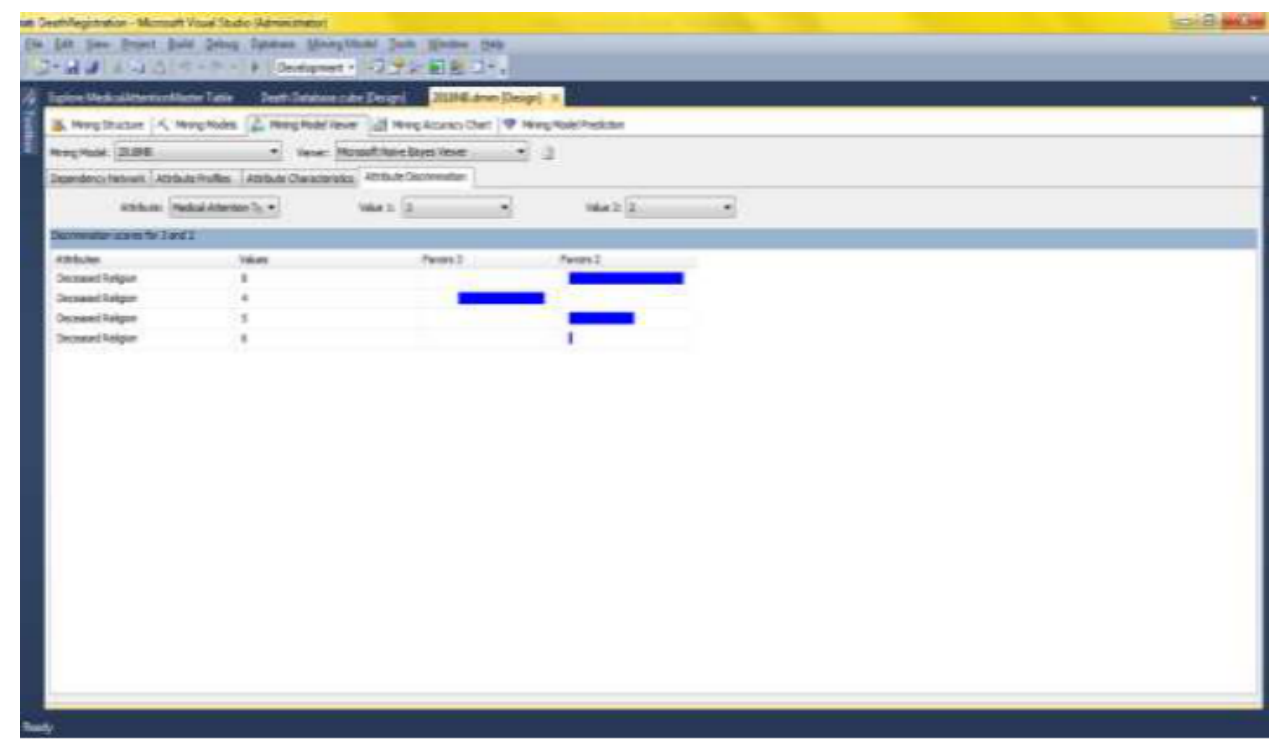

Figure 7 Result for Attribute Discrimination Medical Attention Type 2 and 3

\section{CONCLUSIONS}

The Naïve Bayes algorithm was implemented on Deceased Registration data and some important relationships were derived from the implementation. Furthermore, Naïve Bayes algorithm can also be utilized for Deceased Registration data exploration to provide further insight to the data.

\section{REFERENCES}

[1] Shuo Xu, Bayesian Naïve Bayes classifiers to text classification, Journal of Information Science, SAGA Journals. First Published November 1, 2016, Vol 44, Issue 1, 2018.

[2] Aida Krichene, Using a naive Bayesian classifier methodology for loan risk assessment. Evidence from a Tunisian commercial bank. Journal of Economics, Finance and Administrative Science, vol. 22, no. 42, 2017.

[3] Rajeswari R. P, Kavitha Juliet, Dr.Aradhana, Text Classification for Student Data Set using Naive Bayes Classifier and KNN Classifier, International Journal of Computer Trends and Technology (IJCTT), Volume 43, Number 1, January 2017.

[4] Priyanka Sao, Pro. Kare Prashanthi, E-mail Spam Classification Using Naïve Bayesian Classifier, International Journal of Advanced Research in Computer Engineering \& Technology (IJARCET), Volume 4 Issue 6, June 2015.

[5] Sai Kiran, Jyoti Guru, Rishabh Kumar, Naveen Kumar, Deepak Katariya and Maheshwar Sharma, Credit card fraud detection using Naïve Bayes model based and KNN classifier, International Journal of Advance Research, Ideas and Innovations in Technology, Volume 4, Issue 3, pp.44-48, 2018.

[6] Shruti Bajaj Mangal and Dr. Vishal Goyal, Text News Classification System using Naïve Bayes Classifier, Research Cell: An International Journal of Engineering Sciences, Vol. 3, pp. 209-213. Issue December 2014,

[7] Pallavi Mirajkar and Dr. G. Prasanna Lakshmi, Prediction of Cancer Risk in Perspective of Symptoms using Naïve Bayes Classifier, International Journal of Engineering Research in Computer Science and Engineering, Vol 4, Issue 9, September 2017.

[8] Vrinda and Dr. Komal Kumar Bhatia, Opinion Mining using Naïve Bayes Classifier, International Journal of Engineering Research \& Technology (IJERT), Vol. 6 Issue 04, 2017. 